\title{
LA POLÍTICA DE DESARROLLO RURAL DE LA UNIÓN EUROPEA PARA 2007-2013
}

\author{
Manuel Sáenz Lorite \\ Eugenio Cejudo García \\ Departamento de Geografía Humana \\ Universidad de Granada
}

\section{RESUMEN}

Las actuaciones de desarrollo rural llevadas a cabo en la Unión Europea han adolecido históricamente de una coherencia interna propia y se han ido incorporando al acervo comunitario como respuesta puntual a las necesidades de cada momento. La Política de Desarrollo Rural, que nace como segundo pilar de la PAC tras la aprobación de la Agenda 2000, supuso su formalización como tal pero su diseño, construido por la adicción de medidas dispersas, dista mucho de ser una política internamente construida. La nueva formulación aprobada para 2007-13 la consagra como un apéndice de la PAC, con escasos fondos, con serias incógnitas para su puesta en marcha y desarrollo aunque con una estructura y diseño más lógico y una gestión más simplificada.

Palabras clave: Desarrollo rural, Política Agraria Comunitaria, mundo rural, Unión Europea.

\section{ABSTRACT}

Historically any rural development initiatives undertaken by the European Union have lacked internal coherence and mostly have joined the european legal bodies on a case by case basis. The Rural Development Policy, born as a second basement to the CAP (Common Agriculturan Policy), was a formalizing effort but being designed as a set of sparce initiatives prevented it from succeeding as an internal, solid policy. Under its new definition for the 2007-13 period it becomes an appendix to the CAP, with meager funds and serious issues about its launch and rollout but with an structure and design better suited to its stated aims and a simplified organizing model.

Key words: Rural development, Common Agriculturan Policy, Rural Community, European Union. 


\section{Introducción}

Si se piensa que más de la mitad de la población de los 27 Estados miembro de la Unión Europea (UE) vive en zonas rurales y que éstas cubren el $90 \%$ de su territorio — según criterios OCDE_-, es evidente que el mundo rural y la estrategia que se diseñe para su desarrollo constituye un objetivo político de vital importancia. Por otro lado, la agricultura (incluida la ganadería) y la silvicultura siguen teniendo una importancia capital en la utilización de las tierras y la gestión de los recursos naturales de estas zonas además de constituir una importante plataforma desde la que abordar la necesaria diversificación económica de sus comunidades. Desde esta urgencia ineludible, territorio y patrimonio pasan a formar parte de una misma realidad social, a la que se ha llegado a denominar «patrimonio territorial», en la que el territorio se configura como un producto socialmente construido en el que se congregan elementos de distinta naturaleza creados o modificados, en mayor o menor medida, por el hombre en comunidad y que, legados a través del tiempo, se constituye en seña de identidad de ésta. La gestión tanto de sus recursos naturales como sociales a través de su conservación y puesta en valor se ha convertido en elemento irrenunciable para el desarrollo de las zonas rurales europeas, siendo así reconocido en los últimos años por parte de las propuestas políticas realizadas por la Unión Europea y, lo que es más importante para estos espacios, por los propios ciudadanos europeos: 9 de cada 10 consideran que la agricultura y las zonas rurales son muy importantes para el futuro de Europa (Comisión Europea, 2008: 6)

La Política de Desarrollo Rural de la UE (PDR) ha evolucionado desde un conjunto de actuaciones dirigidas a resolver los problemas estructurales del sector agrícola a una formulación que aborda los desafíos a los que se enfrentan estos espacios atendiendo a las múltiples funciones que la agricultura — para unos - y los territorios rurales — para otros- (Mollard, 2003) desempeñan en una sociedad eminentemente urbana. La nueva realidad rural nos presenta unos territorios en los que a sus funciones productivas, cada vez más diversas y complementarias con las tradicionales agrarias, se añaden otras, no recompensadas por el mercado, relacionadas con la producción de bienes y servicios públicos para la sociedad en su conjunto. Es desde esta óptica desde la que los pagos realizados a los habitantes del mundo rural tendrán justificación y legitimidad social «hay que pasar del acoplamiento a la producción al acoplamiento al territorio» (Moyano, 2007: 18). Esta multifuncionalidad obliga a que las actuaciones que sobre él se quieran acometer requiera de la adopción de criterios y directrices de ordenación territorial que busquen la necesaria complementariedad entre el medio rural y urbano a través de un desarrollo sostenible en su más amplio sentido, pensando en «las necesidades de los habitantes rurales y no, únicamente, de los urbanitas que visitan ocasionalmente estos territorios» (Moltó y Hernández, 2004: 72). En definitiva, un «nuevo paradigma rural» basado en dos principios fundamentales: «la prioridad otorgada al territorio, en lugar de a los sectores, y a la atención prestada a la inversiones, en lugar de a las subvenciones» (OCDE, 2006: 67).

Así lo demuestra, al menos teóricamente, el desarrollo normativo producido en los últimos años. La reforma del primer pilar de la Política Agrícola Común (PAC) realizada en 2003-2004, condujo a la adopción en septiembre de 2005 de la actual configuración de la PDR europea para 2007-2013 ${ }^{1}$, sobre la base de la propuesta de la Comisión de 14 de julio de 2004 (Comisión Europea, 2004a y b). En ella quedan reflejadas las con-

1 Reglamento (CE) no 1698/2005 del Consejo, de 20 de septiembre de 2005, relativo a la ayuda al desarrollo rural a través del Fondo Europeo Agrícola de Desarrollo Rural (DO. L 277 de 21.10.2005).

http://eur-lex.europa.eu/LexUriServ/site/es/oj/2005/1_277/1_27720051021es00010040.pdf 
clusiones de la Conferencia de Salzburgo sobre desarrollo rural (noviembre de 2003) y las orientaciones estratégicas de los Consejos Europeos de Lisboa (2000) y Gotemburgo (2001). A esta evolución nuestro país no ha sido ajeno en la medida en que, por un lado, el mundo rural español supone también el $90 \%$ de nuestro territorio en el que habita el $20 \%$ de la población (35\% si se incluyen las zonas periurbanas). Por otro, la ausencia de una política rural propia que aplicaba — con puntuales actuaciones dignas de mención como Proder - la normativa comunitaria al respecto, se empieza a cubrir con la Ley para el desarrollo sostenible del medio rural, cuyo proyecto se presentó en mayo ${ }^{2}$ y se aprobó el 13 de diciembre ${ }^{3}$ así como la Ley Orgánica que la complementa ${ }^{4}$. No conviene olvidar que la subsidiariedad de los Estados miembro en esta política se impondrá cada vez más en la Europa del siglo XXI.

La finalidad última que se propone este trabajo es la de analizar y valorar la reglamentación que ha sido recientemente aprobada por parte de la Unión Europea sobre la Política de Desarrollo Rural para el periodo de programación 2007-2013. Para ello es necesario enmarcar su actual configuración dentro de la evolución que ésta ha tenido desde sus inicios en los años 70 ligados a la modernización agraria hasta su configuración formal, como segundo pilar de la PAC, con la aprobación de la Agenda 2000. Pasando por su replanteamiento teórico a raíz de la aparición en 1988 de la Comunicación sobre «El futuro del mundo rural» (MAPA, 2002) y las medidas de acompañamiento de la reforma de la PAC de 1992. Por otra parte, la presente estructura del Desarrollo Rural está íntimamente ligada tanto a la reforma de la PAC de 2003-2004 como al nuevo marco financiero para 2007-2013. Una y otro marcados por la ampliación al Este de la UE y por los acuerdos comerciales dentro de la actualmente estancada Ronda Doha de la OMC. Diseño con indudables mejoras en su estructura, diseño y gestión de las actuaciones pero también con heredados vicios al incluirse medidas que se sustentan en planteamientos filosóficos diferentes y exiguos fondos ante los retos que se propone abordar como apéndice de la política de mercados de la PAC.

\section{Los erráticos pasos hacia su actual configuración}

Sin pretender realizar una retrospectiva de los antecedentes de la actual Política de Desarrollo Rural, sí conviene señalar algunos momentos y medidas de especial relevancia para comprender su actual formulación.

\subsection{Los primeros apuntes}

Que en los primeros momentos de la puesta en marcha de la Política Agraria Comunitaria las medidas de carácter socio-territorial estuvieron claramente marginadas en aras del autoabastecimiento agrario a través de la modernización y del incremento de la productividad, ni sorprendió en su momento ni necesita mayor argumentación. Es por ello que se apoyaron las inversiones (capital físico) en las explotaciones agrarias y en el sector de la transformación de sus producciones para mejorar tanto las estructuras agrarias como la competitividad del sector primario. Gradualmente, la atención se desplazó hacia el capital humano, en forma de jubilación anticipada, incorporación de jóvenes agricultores y de formación profesional.

2 http://www.mapa.es/desarrollo/pags/ley/LEY\%20DES.RURAL\%20proyecto\%20(27.4.07).pdf

3 http://www.mapa.es/desarrollo/pags/ley/ley_desrural_14_12_07.pdf

4 http://www.mapa.es/desarrollo/pags/ley/ley_desrural_complementaria14_12_07.pdf 
En los años setenta aparece un primer elemento territorial con la Directiva de áreas de montaña y zonas desfavorecidas ${ }^{5}$ en la que se recogían las diferentes funciones que desempeñaba la agricultura y las dificultades de estas zonas para competir con otros territorios con mayores ventajas naturales y estratégicas. Su finalidad no era otra que detener el éxodo agrícola y rural que amenazaba la supervivencia de ciertas zonas rurales así como la conservación del entorno y el paisaje naturales. Otras actuaciones en este sentido como los Reglamentos socioestructurales ${ }^{6}$ también intentaron ayudar a las áreas rurales a modernizarse y a incrementar su competitividad, aunque con escaso impacto debido básicamente a su limitada dotación presupuestaria.

\subsection{Los primeros cambios: del «futuro del mundo rural» a la «Agenda 2000»}

En los años ochenta se hizo manifiesta la urgencia de prestar una atención específica a las áreas rurales y de diseñar políticas adecuadas a sus necesidades, especialmente al incrementarse los desequilibrios territoriales, básicamente rurales, de forma espectacular tras las incorporaciones producidas en esta década ${ }^{7}$. Es en este contexto en el que se produce, tal y como ya se dijo, la aparición en 1988 de la Comunicación sobre «El futuro del mundo rural», la «Reforma de los Fondos Estructurales» en ese mismo año y la «Reforma de la $P A C »$ de 1992.

Estos tres hechos son esenciales en relación al trabajo que desarrollamos por varios motivos. En primer lugar, porque la Comunicación de 1988 plantea de forma expresa la heterogeneidad y el carácter multifuncional del mundo rural, la necesidad de diversidad de estrategias ante los diferentes problemas ${ }^{8}$ y territorios que lo componen - y que hoy aún se mantienen tanto para nuestro país como en el conjunto de la UE (Molinero, 2002) — así como una metodología para abordarlos participativa e integradora, que tendrá su plasmación en el enfoque Leader. En segundo lugar, porque la reforma de los Fondos Estructurales supuso no sólo una importante inyección financiera para los espacios más deprimidos — la mayor parte rurales - sino también la puesta en marcha de la Iniciativa Comunitaria Leader que, sin lugar a dudas, ha sido el emblema de la PDR europea desde ese momento. Este laboratorio de experiencias — como gusta ser denominada - tiene como objetivo básico el impulso del desarrollo socioeconómico por medio de su diversificación productiva sus-

5 Directiva 268/75 sobre agricultura de montaña y de determinadas zonas desfavorecidas (DO. L. 128 de 19/03/1975).

http://eur-lex.europa.eu/LexUriServ/LexUriServ.do?uri=CELEX:31975L0268:ES:HTML

6 Directiva 159/72 relativa a la modernización de las explotaciones agrarias (DO. L. 96 de 23.4.1972).

http://eurlex.europa.eu/Notice.do?val=27664:cs\&lang=es\&list=27664:cs, 27627:cs, \&pos=1\&page=1\&nbl=2 $\&$ pgs $=10 \&$ hwords $=\&$ checktexte $=$ checkbox $\&$ visu=\#texte

Directiva 160/72 relativa al fomento del cese de la actividad agrícola (DO. L. 96 de 23.4.1972).

http://eur-lex.europa.eu/LexUriServ/LexUriServ.do?uri=CELEX:31972L0160:ES:HTML

Directiva 161/72 relativa a la cualificación profesional de los trabajadores agrícolas. DO L 96 de 23 de abril de 1972 (DO. L. 96 de 23.4.1972).

http://eurlex.europa.eu/Notice.do?val=27666:cs\&lang=es\&list=27666:cs,27734:cs, \&pos=1\&page=1\&nbl=2 \&pgs $=10 \&$ hwords $=\&$ checktexte $=$ checkbox \&visu=\#texte

7 La adhesión de Irlanda, Grecia, España y Portugal incrementaron las diferencias regionales en unos niveles que, salvando las distancias, pueden compararse con los producidos tras la reciente ampliación hacia el Este de la UE. En aquellos años la población activa agraria de éstos oscilaba entre el 20 y $30 \%$ del total.

8 Señala la existencia de tres grades problemas como son la presión del mundo urbano sobre el medio rural -manifestado de forma evidente sobre las zonas próximas a los grandes centros urbanos- que habría que abordar a través de la protección del medio ambiente y la ordenación del espacio rural; la decadencia del mundo rural en su conjunto a combatir con la diversificación productiva y la puesta en valor de su potencial endógeno y, finalmente, los problemas específicos de zonas marginales ligadas a zonas de montaña y equiparables. 
tentándose en un enfoque territorial, la subsidiariedad, la flexibilidad, la participación y la corresponsabilidad de los agentes locales en su propio desarrollo (Esparcia, et al, 2003). En este enfoque, el territorio y la valorización de sus recursos (incluido el capital humano y el capital social) pasan a ser factores estratégicos en la puesta en marcha de acciones de desarrollo sostenible. Por último, la reforma de la PAC de 1992, además del cambio en la filosofía de ayuda al sector agrario, agrupa una serie de actuaciones — denominadas Medidas de Acompañamiento- que van a adquirir cada vez más importancia en relación con la protección medioambiental como elemento vertebrador de la actual política diseñada para estos espacios.

La aceptación generalizada de los principios Leader a pesar de lo limitado de su dotación presupuestaria llevó a F. Fischler a convocar en Cork una Conferencia Europea sobre el Desarrollo Rural (1996). Se defendía la importancia sociocultural del mundo rural, las nuevas demandas que la sociedad le exige, la multifuncionalidad de la agricultura (fundamentalmente en su vertiente de salvaguarda de los recursos naturales y medioambientales) y la necesidad de una nueva reforma de la PAC para adaptarla a la nueva situación económica internacional. Todo ello, reconociendo la importancia del sector agrario como pilar fundamental para el desarrollo rural. Como colofón se aprobó un decálogo con los objetivos que debería marcarse la futura PDR.

La importancia de la Conferencia y de la Declaración aprobada deviene no de la aceptación inmediata de sus propuestas, que no fueron asumidas ante el temor, más que fundado por otra parte, de la reducción de fondos de la política de mercados en beneficio de la de desarrollo rural, cuanto de suponer la primera piedra hacia su actual configuración. La historia de las negociaciones europeas demuestra que difícilmente se aceptan reformas radicales y profundas en las políticas europeas y que todo cambio sustantivo pasa, con anterioridad, por una «tentativa frustrada» que prepara el camino para su posterior implantación algo suavizada en sus planteamientos iniciales.

\subsection{La formalización de la política de desarrollo rural: la Agenda 2000}

A mediados de los años noventa la UE contaba con una serie de instrumentos para responder a los objetivos de reestructuración agrícola, desarrollo territorial/local e integración medioambiental — tal y como ya se ha señalado de forma muy sucinta— pero no disponía, ni real ni formalmente, de una política de desarrollo rural como tal (Delgado, 2004: 259). Con la aprobación de la llamada Agenda 2000 (Comisión Europea, 1997) en el Consejo de Berlín de $1999^{9}$ se produce la aparición formal de dicha política dentro del acervo comunitario al establecerse que la PAC, a partir de entonces, se sustentaría en dos «pilares»: el primero orientado a la política de mercados, el segundo al desarrollo rural.

Este segundo pilar de la PAC se articuló en torno a dos tipos de actuaciones. Por un lado, la aprobación del Reglamento $1257 / 99^{10}$ sobre Desarrollo Rural y el 1259/9911 de

9 En él se fijaron, además, las Perspectivas Financieras para el presupuesto de la UE durante los años 2000-2006. http://www.consilium.europa.eu/ueDocs/cms_Data/docs/pressData/es/ec/00100.es9.html

10 Reglamento (CE) n $n^{\circ}$ 1257/1999 del Consejo, de 17 de mayo, sobre la ayuda al desarrollo rural a cargo del Fondo Europeo de Orientación y de Garantía Agrícola (FEOGA) y por el que se modifican y derogan determinados Reglamentos (DO. L 160 de 26.6.1999).

http://eur-lex.europa.eu/LexUriServ/site/es/oj/1999/1_160/1_16019990626es00800102.pdf

11 Reglamento (CE) n $\mathrm{n}^{\circ}$ 1259/99 del Consejo, de 17 de mayo, por el que se establece las normas comunes para los regímenes de ayuda directa en el marco de la política agrícola común que se aplicarán a los pagos abonados directamente a los agricultores (DO. L 160 de 26.6.1999).

http://eur-lex.europa.eu/LexUriServ/site/es/oj/1999/1_160/1_16019990626es01130118.pdf 
carácter horizontal, ambos de aplicación general para el territorio europeo, y, por otro, la puesta en marcha de la iniciativa Leader $+{ }^{12}$ y Proder $\mathrm{II}^{13}$ en clara sintonía y evolución con las predecesoras Leader I y II así como Proder I, respectivamente. Estamos con este desarrollo normativo ante la formalización de una política de Desarrollo Rural, concebida como una parte muy menor de la PAC pero que adquiere carta de naturaleza política y jurídica dentro de la UE.

Pero que se «visualice» normativamente una política no lleva implícito, necesariamente, que se formule y desarrolle como tal.

En efecto, los Reglamentos antes citados han tratado de integrar una serie de medidas en un plan único, y discutiblemente coherente, a través de 4 objetivos básicos: la creación de unos sectores agrícola y forestal más fuertes; la mejora de la competitividad de las zonas rurales y el mantenimiento y preservación de un medio ambiente y un patrimonio rural único. Ahora bien, no han supuesto la introducción de ninguna novedad real, se han limitado a recoger una relación de 22 medidas, todas ya existentes y dispersas por diferentes normativas, para que los Estados miembro escojan aquéllas que mejor se adecúen a sus zonas rurales.

Por otra parte, su asignación presupuestaria es la suma de las partidas que se contemplaban en cada una de las diferentes medidas por separado sólo que reunificadas bajo una misma denominación. Además el hecho de ser considerada como «segundo pilar de la PAC» le confiere un fuerte sesgo sectorial y agrario al ser «reubicadas» bajo una nueva denominación. Estamos, por tanto, ante las mismas medidas «cambiadas» de sitio con una escasa asignación financiera dentro de la PAC (30 frente a los casi 300 millardos de euros del primer pilar de la PAC) dirigidas mayoritariamente a los agricultores — que pese a su importancia son una clara y creciente minoría - y no a toda la población que habita estos espacios rurales, como debería haberse contemplado. Nada más rotundo que lo establecido en el art. 2 del Reglamento 1257/99: "Las medidas de desarrollo rural acompañarán y servirán de complemento a otros instrumentos de la política agrícola común (...)». Que en el art. 33 del capítulo IX «Fomento de la adaptación y desarrollo de las zonas rurales» de la citada normativa aparezca entre uno de los 13 objetivos que se señalan —en los que aparecen mezclados aspectos tan dispares como la «reparcelación de tierras», «la comercialización de los productos agrarios de calidad», «la ingeniería financiera», «la gestión de los recursos hídricos agrícolas», etc.,— «la renovación y desarrollo de los pueblos y la protección y conservación del patrimonio rural» supone más la plasmación de una idea marginal que una verdadera estrategia rural.

Ahora bien cuestionando, como lo hacemos, la enorme desproporción existente entre los fondos asignados a uno y otro pilar, lo que entendemos incuestionable es que no se puede convertir al agricultor en subsidiado ambientalista. Las externalidades positivas que su actividad y los territorios rurales generan para el conjunto de la sociedad deben ser recompensadas y complementar las rentas obtenidas a través del desarrollo de la actividad agraria, entendida como piedra angular del desarrollo rural (Molinero, 2006: 104-106) orientada a la obtención de producciones de calidad cada vez más demandadas (Armesto, 2007).

12 Comunicación de la Comisión a los Estados miembro, de 14 de abril de 2000, por la que se fijan orientaciones sobre la Iniciativa Comunitaria de desarrollo rural (Leader+) (DO. C 139 de 18.5.2000).

http://eur-lex.europa.eu/LexUriServ/site/es/oj/2000/c_139/c_13920000518es00050013.pdf

13 Real Decreto 2/2002, de 11 de enero, por el que se regula la aplicación de la Iniciativa Comunitaria Leader+ y los programas de desarrollo endógeno de grupos de acción local incluidos en los Programas Operativos Integrados y en los Programas de Desarrollo Rural (PRODER) (BOE nº 11 de 12 de enero). http://www.mapa. es/desarrollo/pags/LEGISLACION/leader/RD2-2002.pdf 
Por su parte, la nueva iniciativa comunitaria Leader+ - convertida ya en «todo un fenómeno mediático, social, político y, en parte, también económico» (Esparcia, 2006: 8889)_ hace un especial hincapié en recuperar la función de laboratorio, establecido en Leader I, a través de propuestas que deberán demostrar su carácter piloto, reforzando el interés de la cooperación y del trabajo en red de los territorios rurales. Este intento de recuperar antiguos valores está en clara relación con la institucionalización y burocratización en la que se encontraban los grupos de desarrollo, enfrascados más en funciones administrativas que dinamizadoras. Por otro lado, la generalización de todo el espacio rural europeo como «territorio elegible», aunque puede suponer una ventaja para muchos espacios, conduce a que se vean más favorecidas las zonas rurales de territorios de mayor nivel de desarrollo al estar en mejores condiciones de establecer fórmulas de partenariado y de gestión más viables y competitivas que las más desfavorecidas. Finalmente, señalar la obligatoriedad de que los Grupos de Desarrollo estén compuestos por los diferentes sectores socioeconómicos del territorio, debiendo representar los agentes económicos y las asociaciones, como mínimo, un $50 \%$ en los órganos de decisión.

Pero el elemento que más nos interesa resaltar en este trabajo es que Leader + plantea dar un salto desde las acciones innovadoras de Leader II a los espacios innovadores situando el territorio como elemento central de las estrategias de desarrollo de los diferentes grupos. El territorio ha de ser considerado como un todo aglutinador de los diferentes recursos existentes a la vez que valor añadido en las diferentes estrategias de calidad e innovación capaces de pone en valor estos recursos. Estas estrategias de desarrollo deben articularse en función de un aspecto dominante - representativo de su identidad, sus recursos o sus conocimientos técnicos específicos - que sea capaz de ejercer de aglutinador de integrantes y proyectos a desarrollar. En definitiva, como establece el apartado 8 de la Comunicación sobre Leader + , su objetivo es «incitar y ayudar a los agentes del mundo rural a reflexionar sobre el potencial de su territorio, en una perspectiva más a largo plazo, y el fomentar la aplicación de estrategias originales de desarrollo sostenible, integradas, de calidad y destinadas a la experimentación de nuevas formas de valorización del patrimonio natural y cultural, de mejora del entorno económico, a fin de contribuir a la creación de empleo y de mejora de la capacidad de organización de las respectivas comunidades». Estamos, a nuestro entender, ante la actuación en la que con mayor claridad se concreta el maridaje, del que antes hablábamos, entre desarrollo rural y patrimonio territorial a través de la gestión y puesta en valor de diferentes recursos naturales y culturales que conforman éste. Como bien señala Plaza (2006: 84) «el futuro de un territorio rural puede depender de su capacidad para utilizar la herencia de su pasado en el momento oportuno. La valorización del patrimonio supone un componente ineludible para el proceso de desarrollo local».

\subsection{Reforma de la PAC de 2003 y la Conferencia de Salzburgo}

Dos elementos más sirven para comprender la configuración actual de la Política de Desarrollo Rural europea. De un lado, la reforma de la PAC que se instaura a partir de 2003-2004 ${ }^{14}$ en la medida en que incrementa el carácter complementario, financieramente

14 Se omiten los Reglamentos de aplicación a las diferentes Organizaciones Comunes de Mercado.

Reglamento (CE) $n^{\circ}$ 1782/2003 del Consejo, de 29 de septiembre, por el que se establecen disposiciones comunes aplicables a los regímenes de ayuda directa en el marco de la política agrícola común y se instauran determinados regímenes de ayuda a los agricultores.

http://eur-lex.europa.eu/LexUriServ/site/es/consleg/2003/R/02003R1782-20060101-es.pdf 
hablando, de los dos pillares de la PAC — hasta este momento poco interconectados - a través de la transferencia de fondos del primer al segundo pilar por medio de la modulación de las ayudas, de su condicionalidad al cumplimiento de ciertos requisitos medioambientales así como por la implantación de nuevas medidas relativas a la calidad de los alimentos y el bienestar de los animales. En definitiva, se refuerza de forma clara el carácter multifuncional de la agricultura a la vez que se dota al desarrollo rural de más fondos, al menos teóricamente como luego desarrollaremos.

De otro, la celebración en noviembre de 2003 de la II Conferencia Europea sobre Desarrollo Rural en Salzburgo bajo el lema «Sembrar las semillas del futuro rural. Perspectivas de la política rural en una Europa ampliada». Las conclusiones adoptadas establecen los principios que inspiran la actual Política de Desarrollo Rural europea:

1. Un campo vivo no sólo redunda en beneficio de la sociedad rural sino también en el de la sociedad en su conjunto.

2. La preservación de la diversidad del campo europeo y el estímulo de los servicios que puede prestar la agricultura multifuncional revisten cada vez mayor importancia.

3. La competitividad del sector agrario debe ser un objetivo fundamental.

4. Debe aplicarse en todas las zonas rurales de la Unión Europea ampliada para hacer frente a la reestructuración del sector agrícola y la evolución del comercio internacional.

5. Debe responder a las necesidades de la sociedad rural en general y contribuir a su cohesión.

6. Debe llevarse a cabo mediante la colaboración de las organizaciones públicas y privadas y la sociedad civil, de acuerdo con el principio de subsidiariedad.

7. Se debe dar mayor responsabilidad a las asociaciones de colaboración de los programas para la formulación y aplicación de sus estrategias generales.

8. Es necesario y urgente efectuar su simplificación a través de en un sistema único de programación, financiación y control.

Finalmente, y en el contexto de las nuevas Perspectivas Financieras para el período de programación 2007-2013, la Comisión Europea llevó a cabo un análisis en profundidad de la PDR, que incluye una Evaluación de Impacto Ampliada (Comisión Europea, 2005) (5 $^{15}$ de la futura política de desarrollo rural. Las conclusiones de esta Evaluación y de la citada Conferencia se reflejan en un nuevo borrador de Reglamento sobre desarrollo rural presentado, como ya se dijo, en julio de 2004.

Reglamento (CE) n $n^{\circ} 796 / 2004$ de la Comisión, de 21 de abril, por el que se establecen disposiciones para la aplicación de la condicionalidad, la modulación y el sistema integrado de gestión y control previstos en el Reglamento (CE) $\mathrm{n}^{\circ}$ 1782/2003 del Consejo.

http://eur-lex.europa.eu/LexUriServ/site/es/consleg/2004/R/02004R0796-20050325-es.pdf

Reglamento (CE) n 795/2004 de la Comisión, de 21 de abril, que establece disposiciones de aplicación del régimen de pago único previsto en el Reglamento (CE) $n^{\circ} 1782 / 2003$ del Consejo.

http://eur-lex.europa.eu/LexUriServ/site/es/consleg/2004/R/02004R0795-20050101-es.pdf

Reglamento (CE) no 118/2005 de la Comisión, de 26 de enero, que modifica el anexo VIII del Reglamento (CE) no 1782/2003 del Consejo y establece límites presupuestarios para la aplicación parcial o facultativa del régimen de pago único y las dotaciones financieras anuales del régimen de pago único por superficie establecidos en el citado Reglamento.

http://eur-lex.europa.eu/LexUriServ/site/es/consleg/2005/R/02005R0118-20050416-es.pdf

15 Dicha Evaluación se recoge en SEC (2005) 914 que actualiza otra realizada el año anterior SEC (2004) 931. Los SEC son documentos internos vinculados a los procesos de toma de decisiones y al funcionamiento general de los servicios de la Comisión. 


\subsection{Balance de resultados}

En modo alguno se pretende aquí realizar un exhaustivo recorrido por los aspectos favorables, unos, y discutibles, otros, que han jalonado las actuaciones que brevemente hemos ido comentando. Pero sí queremos hacer algunas valoraciones que nos parecen oportunas.

Con la perspectiva que da los 40 años de evolución de la PAC, se puede afirmar que la preocupación por el mundo rural se han ido acrecentando tanto en la importancia de las actuaciones llevadas a cabo, en su dotación financiera o en su filosofía, de marcado carácter sectorial en sus inicios allá por los años 70 a un componente más integrado y ruralista en los primeros años del siglo XXI.

En este largo transcurrir los cambios acontecidos con la reforma de los Fondos Estructurales en 1988 -incremento de fondos, diseño por objetivos, nuevas fórmulas de participación, Iniciativa Leader, etc_ y la de la PAC de 1992 — pagos compensatorios, medidas de acompañamiento, etc.—, inspirada en la Comunicación sobre «el futuro del mundo rural»- suponen un cambio de rumbo, sin posibilidad de marcha atrás, en la consideración de la necesidad de formular una Política de Desarrollo Rural europea sustentada en algunos de los principios en los que se inspiraron las reformas antes citadas. Ya se han comentado brevemente pero razones de orden interno y externo abocaron a este cambio (Sáenz, et al, 2000; Cejudo y Maroto, 2000). La aparición formal de dicha política dentro del acervo comunitario tras la aprobación del la Agenda 2000 como segundo pilar de la PAC es el punto culminante de este largo recorrido.

Así las cosas, ¿se puede entender que Europa dispone de una política de Desarrollo rural como tal tras la Agenda 2000? A nuestro entender, no. Los Reglamentos que regulan la denominada Política de Desarrollo Rural son el resultado de la compilación bajo una única denominación de una serie de medidas (adoptadas en momentos diferentes para resolver problemas concretos) con lógicas muy diversas - cuando no antagónicas - que intenta dar respuesta a los retos financieros, políticos e internacionales de una Europa que se amplía y a unas nuevas negociaciones internacionales dentro de la OMC que se están empezando a acometer. En este momento $-\mathrm{y}$ viene siendo ya costumbre - como en la mayoría de las ocasiones las declaraciones y buenas intenciones no se han traducido en medidas, instrumentos y financiación suficiente.

Esta histórica falta de coherencia en las actuaciones de desarrollo rural, grave en sí misma, ha conllevado que existan serias dudas sobre su capacidad para resolver eficazmente los problemas del mundo rural. Ello al menos por dos motivos. Uno de carácter económico, otro ligado a la defensa de la multifuncionalidad de la actividad agraria y de los espacios rurales como vertebradora de sus territorios.

El primer aspecto es fácilmente entendible si se tiene en cuenta que los fondos directamente ligados a esta política no han superado el $10 \%$ del presupuesto comunitario. De hecho representaron el 8,74\% en 2006, último año del anterior periodo de programación. Este dato debe ser puesto en relación, al menos, con otros dos. El raquítico presupuesto con el que cuenta la UE que en ningún caso, en término de gasto real, superó el 1,14\% de la RNB de la UE - ya con 25 miembros- durante el periodo 2000-2006. Dicho valor se encuentra a abismal distancia respecto de los niveles presupuestarios que serían necesarios mantener para generar «impactos relevantes a escala europea en la provisión de bienes públicos europeos, la estimulación del crecimiento sostenible o promover la cohesión social y territorial que debería oscilar, según autores, entre 5-7\% del PIB» (Etxezarreta, et al, 2005, 29). A ello se une — dejando a un lado la propia dinámica del mercado- el efecto desequilibrador que desde el punto de vista social y territorial genera la propia PAC en la medida en que sus 
sucesivas reformas en nada han modificado tal capacidad a través del denominado primer pilar, el mejor dotado financieramente desde su nacimiento (Cejudo, 2001).

Si todo lo dicho no fuese suficiente, el Tribunal de Cuentas (2006) en un informe especial realizado sobre la PDR aplicada en el UE durante el periodo 2000-2006 obtiene las algunas conclusiones demoledoras:

- Una parte importante de las inversiones en desarrollo rural se han ejecutado en zonas que no son predominantemente rurales por lo que, en relación con el periodo de programación anterior, las realizadas en zonas rurales han disminuido considerablemente

- Se ha carecido de condiciones efectivas y de procedimientos de selección para canalizar la financiación hacia las zonas geográficas y los beneficiarios más necesitados.

- Los objetivos perseguidos han sido demasiado generales y faltan estrategias para aplicar dicha política.

- Los programas de los Estados miembro se concentra en gran media en el sector agrícola y no tienen suficientemente en cuenta las características de la zona geográfica destinataria de la ayuda.

El argumento de la multifuncionalidad también se encuentra en entredicho, no tanto por su sustento teórico que defendemos, cuanto por su utilización por parte de la Unión Europea como estrategia de negociación política dentro del marco de las negociaciones multilaterales de la OMC. Es evidente que la implantación de la condicionalidad a la hora de recibir cualquier ayuda directa supone el reconocimiento de la multifuncionalidad y con ello de la producción de bienes y servicios de interés general que deberán ser recompensados socialmente. Multifuncionalidad que, además de ser un concepto aquejado de una cierta ambivalencia — «intencionadamente ambiguo y polisémico en la medida en que no se explicita si de lo que se habla es de la actividad agraria como sector o de los espacios rurales en tanto territorios; o si cuando ésta se aborda se hace desde un enfoque positivo o normativo» (Moyano y Garrido, 2006: 73) —, ha sido usada como elemento estratégico de negociación dentro de la OMC utilizándose o desapareciendo de los documentos oficiales de la UE según se desarrolla dicho proceso. De hecho, tras el rechazo que supuso su propuesta dentro de la agenda de las negociaciones de la Ronda Doha, el término se abandona en documentos tan significativos como los de la Conferencia de Salzburgo o sólo aparece una vez y en los textos de la reforma de 2003 (Díez y Trueba, 2006: 52).

En este panorama sombrío destaca, por méritos propios, la Iniciativa Comunitaria Leader y el Programa Proder. No en vano este último puede ser considerado como «la primera política española de desarrollo de áreas rurales con enfoque territorial e integrado» tanto en su primera versión como Programa Operativo como en la segunda en la que «sus fondos se han incrementado sustancialmente y su formulación se ha traducido en un paquete de medidas de desarrollo endógeno incluidas en los Programas Operativos Integrados de Objetivo 1 así como en los Programas de Desarrollo Rural de fuera de Objetivo 1» (MAPA, 2003: 644 y 659). Es cierto que no están exentos de muchas críticas que parten de la discutible credibilidad de los datos que aportan algunos Informes finales (Navarro, 2007), pasando por los problemas derivados de una novedosa metodología de trabajo, la desconfianza de ciertos sectores y agentes de la sociedad rural - los agricultores y sus asociaciones son el ejemplo más evidente-, la politización de la Grupos encargados de llevar a cabo dichos programas o la utilización de sus fondos por parte de los ayuntamientos para cubrir, ante la ausencia de iniciativa privada, lícitamente necesidades que sus exiguos presupuestos impiden (Alario y Baraja, 2006: 291). Ahora bien, su indiscutible valoración positiva general debe 
ser entendida más en términos cualitativos que cuantitativos —en muchos casos menor que la esperada (Frutos, Hernández y Ruíz, 2006: 265) y concentrada en los núcleos ya de por sí más dinámicos (Cejudo y Navarro, 2003) — y ser vista más como camino a seguir que como realidad alcanzada en busca de los «beneficios y sinergias que en términos sociales y económicos puedan ser capaces de generar dichas inversiones en el medio y largo plazo» (Márquez et al, 2006: 312-313).

\section{El nuevo diseño de la política de desarrollo rural}

La nueva Política de Desarrollo Rural de la UE ve la luz, como ya se dijo, con la promulgación del Reglamento (CE) 1698/2005 así como del 1974/2006 que desarrolla sus disposiciones de aplicación ${ }^{16}$. Bajo un sólo fondo se ofrecen un conjunto de medidas, antes dispersas entre las políticas sectoriales y territoriales, con el fin de ofrecer una mayor transparencia, simplicidad y coherencia en relación con las existentes anteriormente.

\subsection{Objetivos y enfoque estratégico}

Los objetivos fundamentales que se establecen en este nuevo Reglamento se expresan en su art.4 y están en sintonía con la mayor parte de las conclusiones de la Conferencia de Salzburgo, del Consejo Europeo de Lisboa — que enfatiza el objetivo de convertir a la UE en una zona altamente competitiva a través de I+D+i-o de Gotemburgo — que se centra en la protección del medio ambiente y el desarrollo sostenible- y de los objetivos de las Perspectivas Financieras para 2007-2013. Éstos son:

1. Mejorar la competitividad de la agricultura y la silvicultura mediante la ayuda a la reestructuración, el desarrollo y la innovación.

2. Mejorar el medio ambiente y el medio rural por medio de la gestión de las tierras.

3. Mejorar la calidad de vida de las zonas rurales y fomentar la diversificación de la actividad económica.

Estos objetivos se aplicarán a través de cuatro Ejes, los tres primeros corresponden a cada uno de los tres objetivos antes señalados a los que se suma un cuarto de carácter «transversal o metodológico»: el Eje Leader.

Para garantizar una estrategia equilibrada, se establece una financiación comunitaria mínima para cada uno de los Ejes temáticos. Los porcentajes propuestos, del 10, 25 y 10 para los Ejes 1, 2 y 3 respectivamente, garantizan que cada programa refleje al menos los tres objetivos políticos principales a la vez que permite a los Estados miembro - y a sus regiones - primar su situación y necesidades particulares. Para el Eje Leader se reserva un mínimo del $5 \%$ (2,5\% para los nuevos Estados miembro) de financiación comunitaria y sus gastos se aplican a los tres Ejes temáticos. Con este enfoque se pretende conjugar unas prioridades comunitarias en el ámbito europeo con la flexibilidad estatal y regional para el diseño final de su propia política, en busca del necesario equilibrio entre la dimensión sectorial (reestructuración agrícola) y la dimensión territorial (gestión de las tierras y desarrollo socioeconómico de las zonas rurales), en sus respectivos territorios. La nueva estructura queda claramente reflejada en la figura $n^{\circ} 1$.

16 Reglamento (CE) no 1974/2006 de la Comisión de 15 de diciembre (DO. L 368 de 23.12.2006).

http://eur-lex.europa.eu/LexUriServ/LexUriServ.do?uri=OJ:L:2006:368:0015:0073:ES:PDF 

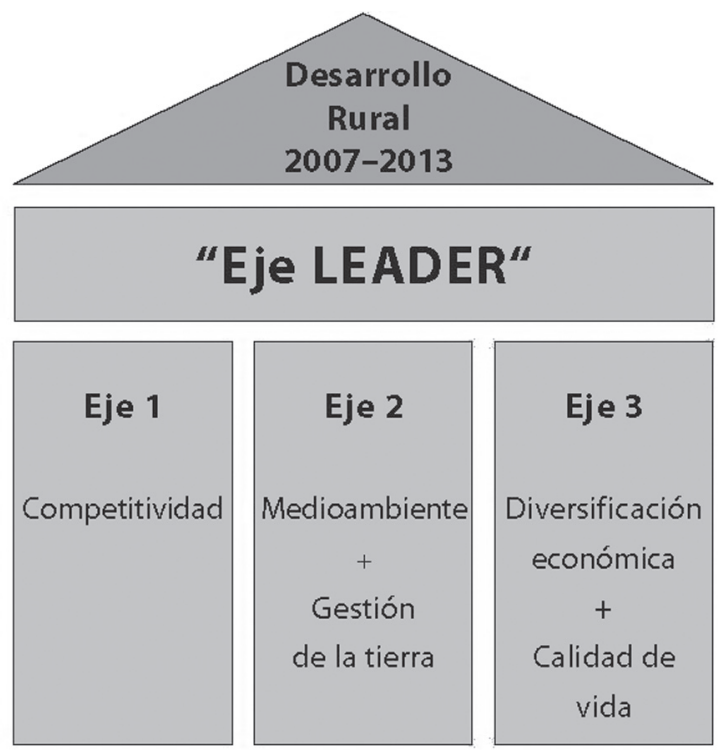

\section{Un único conjunto de normas de programación, financiación, control y auditoría}

\section{Un único Fondo de Desarrollo Rural}

Figura 1. Estructura del Desarrollo Rural 2007-13.

Fuente: Imagen capturada de la web http://ec.europa.eu/agriculture/rurdev/index_es.htm

La elaboración, como ya se dijo, por parte del Consejo de unas Directrices Estratégicas en esta materia refuerza de forma clara el enfoque estratégico de las actuaciones a llevar a cabo (Títulos II y III). En base a ellas cada Estado miembro elabora un Plan Estratégico Nacional en el que recoge sus prioridades de actuación así como la participación del FEADER. Éste se concreta a través de Programas de Desarrollo Rural que contienen un conjunto de medidas agrupadas en torno a los diferentes Ejes así como su financiación. Son las siguientes:

1. Mejorar la competitividad de los sectores agrícola y silvícola sobre la base de su enorme capacidad productiva y de la obtención de producciones de calidad con creciente demanda europea y mundial. Para ello es necesario potenciar la transferencia de conocimientos, la modernización, innovación y calidad de la cadena alimentaria en los sectores prioritarios así como la inversión en capital físico y humano.

2. Mejorar el medio ambiente y el paisaje rural. La protección y mejora de los recursos naturales y paisajísticos de las zonas rurales de la UE debe contribuir al logro de tres prioridades de la UE: la biodiversidad y la preservación de los sistemas agrícolas y silvícolas de elevado nivel natural así como de los paisajes agrícolas tradicionales, el régimen hidrológico y el cambio climático. 
3. Mejorar la calidad de vida de las zonas rurales y el fomento de la diversificación. La creación de oportunidades de empleo y la mejora de la calidad de vida son básicas para que las zonas rurales sigan siendo atractivas para las generaciones futuras. Se deberá prestar especial atención a las necesidades específicas de las mujeres, los jóvenes y los trabajadores de más edad.

4. Desarrollar la capacidad local de creación de empleo y diversificación. La capacidad de gestionar desde lo local favorece no sólo la mejora de la gobernanza sino también el mejor aprovechamiento del potencial de desarrollo endógeno de las zonas rurales.

5. Garantizar la coherencia de la programación. Los Estados miembro en el diseño de su propia estrategia deberán velar porque exista la máxima sinergia posible entre los diferentes Ejes y dentro de éstos así como imbricarla con otras actuaciones y políticas prioritarias de la UE.

6. Complementariedad entre instrumentos comunitarios. Los Estados miembro deberán velar por la complementariedad y coherencia de las acciones que vayan a financiar el FEDER, el FSE y el FAEDER, en un territorio concreto y en un ámbito de actividad determinado.

En definitiva, se establecen cuatro Directrices Estratégicas en concordancia y para la aplicación de los cuatro Ejes en los que se estructuran las medidas de la Política de Desarrollo Rural europea y otras dos que refuerzan aspectos básicos en este nuevo diseño: la coherencia en la programación y la complementariedad en cuanto a los instrumentos para llevarla a cabo.

\subsection{Las medidas de desarrollo rural contempladas}

Para cada Eje estará disponible una serie de medidas a las que los Estados miembro podrán acogerse en sus diferentes Programas de Desarrollo Rural, elaborados a nivel nacional o regional, según sus prioridades y estrategias (se resumen en el cuadro $\mathrm{n}^{\circ} 1$ ).

\subsubsection{Medidas del Eje 1}

A pesar de que la agricultura esté perdiendo presencia como actividad principal en un número creciente de zonas rurales, sigue desempeñando un papel determinante en lo que respecta a la gestión del territorio de la UE, no sólo como productora de alimentos sino también de bienes y servicios públicos. Por otro lado, es necesario incrementar su competitividad ante la creciente liberalización del comercio agrícola mundial marcado por el papel que las transnacionales desempeñan en lo que Etxezarreta denomina «tercer régimen agroalimentario» (2006: 51-56).

Las medidas incluidas en este Eje para hacer frente a estos desafíos se clasifican en cuatro grupos:

- Recursos humanos. Se plantea la instalación de jóvenes agricultores, la jubilación anticipada de empresarios y trabajadores agrarios así como la formación e información a través de servicios de asesoramiento a las explotaciones agrícolas.

- Potencial físico. La UE apoya la modernización de las explotaciones agrícolas, la valorización de los productos agrícolas y forestales a través de su transformación y comercialización, la elaboración de nuevos productos y procesos innovadores y de calidad así como de la mejora de las infraestructuras relacionadas con el desarrollo y adaptación de la agricultura y silvicultura. 


\section{Cuadro $\mathrm{N}^{\circ} .1$.}

SINTESIS DE LA POLITICA DE DESARROLLO RURAL DE LA UE 2007-2013

\begin{tabular}{|c|c|c|}
\hline \multicolumn{2}{|l|}{ Fijación de objetivos } & $\begin{array}{l}\text { Estrategia de la UE } \\
\text { Estrategia nacional } \\
\text { Programas de DR }\end{array}$ \\
\hline \multirow[t]{5}{*}{$\begin{array}{l}\text { Eje } n^{0} 1 \\
\text { Mejora de la } \\
\text { competitividad del } \\
\text { sector agrícola y } \\
\text { silvícola }\end{array}$} & \multirow[t]{2}{*}{ medidas } & $\begin{array}{l}\text { Recursos humanos: } \\
\text { Acciones de información y formación profesional } \\
\text { Jóvenes agricultores } \\
\text { Jubilación anticipada } \\
\text { Utilización de servicios de asesoramiento } \\
\text { Implantación de servicios de gestión, sustitución y asesoramiento de las } \\
\text { explotaciones agrícolas } \\
\text { Capital físico: } \\
\text { Inversiones modernización explotaciones y mejora valor silvicultura } \\
\text { Transformación, comercialización, cooperación para la innovación } \\
\text { Infraestructura agrícola y forestal } \\
\text { Recuperación del potencial de producción agrícola } \\
\text { Calidad de la producción y productos agrícolas } \\
\text { Ayuda temporal cumplimiento de normas } \\
\text { Régimen para incentivar la calidad de los alimentos } \\
\text { Promoción de la calidad de los alimentos }\end{array}$ \\
\hline & & $\begin{array}{l}\text { Medidas transitorias: } \\
\text { Semisubsistencia } \\
\text { Creación de agrupaciones de productores }\end{array}$ \\
\hline & porc de financiación & mínimo $10 \%$ \\
\hline & porc cofinanciación UE & máximo $50-75 \% *$ \\
\hline & aplicación territorial & todas las zonas rurales \\
\hline \multirow[t]{5}{*}{$\begin{array}{l}\text { Eje } n^{\circ} 2 \text { Mejora del } \\
\text { medio ambiente y del } \\
\text { espacio rural }\end{array}$} & medidas & $\begin{array}{l}\text { Utilización sostenible de las tierras agrícolas: } \\
\text { ZMF de montaña } \\
\text { Otras zonas con desventajas } \\
\text { Zonas agrícolas Natura } 2000 \\
\text { Agroambiente / bienestar animal (obligatoria) } \\
\text { Ayuda a inversiones no productivas } \\
\text { Utilización sostenible de las tierras forestales } \\
\text { Forestación (tierras agrícolas y no agrícolas) } \\
\text { Agrosilvicultura } \\
\text { Zonas forestales Natura } 2000 \\
\text { Medioambiente forestal } \\
\text { Recuperación de potencial forestal } \\
\text { Ayuda a inversiones no productivas }\end{array}$ \\
\hline & base de ref (agric) & condicionalidad \\
\hline & porc de financiación & mínimo $25 \%$ \\
\hline & porc cofinanciación UE & máximo 55/80\%* \\
\hline & aplicación territorial & todas las zonas rurales \\
\hline \multirow[t]{4}{*}{$\begin{array}{l}\text { Eje no } 3 \text { Calidad } \\
\text { de vida en el } \\
\text { medio rural y } \\
\text { diversificación de la } \\
\text { economía rural }\end{array}$} & medidas & $\begin{array}{l}\text { Calidad de vida: } \\
\text { Servicios básicos para la economía y población rural (creación e } \\
\text { infraestructuras) } \\
\text { Renovación y desarrollo de pueblos } \\
\text { Protección y conservación del patrimonio rural } \\
\text { Diversificación económica: } \\
\text { Diversificación hacia actividades no agrícolas } \\
\text { Ayuda a las microempresas } \\
\text { Promoción de actividades turísticas } \\
\text { Formación, adquisición de capacidades y promoción: } \\
\text { Formación e información } \\
\text { Adquisición de capacidades, promoción y aplicación }\end{array}$ \\
\hline & porc de financiación & mínimo $10 \%$ \\
\hline & porc cofinanciación UE & máximo 50/75 \%* \\
\hline & aplicación territorial & todas las zonas rurales \\
\hline \multirow{4}{*}{ Eje Leader } & aplicación & $\begin{array}{l}\text { Enfoque Leader para territorios seleccionados en virtud de los } 3 \text { Ejes } \\
\text { temáticos }\end{array}$ \\
\hline & porc de financiación & mínimo $5 \%$ (25\% en los nuevos Estados miembro) \\
\hline & porc cofinanciación UE & máximo 55/80 \%* \\
\hline & aplicación territorial & todas las zonas rurales, territorios seleccionados \\
\hline
\end{tabular}

*El primer porcentaje de cofinanciación se refiere a todas las regiones, con excepción de las de Convergencia; el segundo se aplica a ellas.

FUENTE: Reglamento (CE) 1968/2005 del Consejo de 20 de septiembre de 2005. Elaboración propia. 
- Calidad. En el ámbito de la calidad alimentaria existen dos medidas básicas. Por un lado, incentivos económicos a los agricultores que participen voluntariamente en los programas nacionales o comunitarios de mejora de la calidad de los productos agrícolas y de sus métodos de producción (denominaciones de origen, producción ecológica, etc). Por otro, ayudas a las agrupaciones de productores que realicen actividades de información a los consumidores y de promoción de los productos de calidad así como una ayuda temporal y decreciente a los agricultores que quieran cumplir estas normas de calidad.

- Medidas transitorias para los nuevos Estados miembro en apoyo a la agricultura de semisubsistencia así como a la creación y funcionamiento de agrupaciones de productores.

\subsubsection{Medidas del Eje 2}

Las medidas contempladas en este Eje, además de estar sujetas al cumplimiento por parte de los beneficiarios de los requisitos que marca la condicionalidad, pretenden garantizar la oferta de servicios medioambientales — a través de medidas agroambientales en las zonas rurales - y la gestión sostenible de la tierra (en particular en zonas con desventajas físicas y naturales) con el fin de preservar y mejorar el espacio y el paisaje natural. Las actividades cofinanciadas deberán centrarse en prioridades como la lucha contra el cambio climático, la promoción de la biodiversidad, la calidad del agua o la disminución del riesgo y del impacto de catástrofes naturales.

Se clasifican en dos capítulos:

- Utilización sostenible de las tierras agrícolas. Los agricultores desempeñan un papel esencial en la prestación de servicios medioambientales y, por consiguiente, pueden efectuarse pagos a los agricultores que asuman voluntariamente compromisos agroambientales así como de bienestar animal por un período mínimo de cinco años. Se contemplan también pagos anuales para compensar a los agricultores por las pérdidas de ingresos resultantes de la aplicación de la red Natura $2000^{17}$ y de la Directiva Marco del Agua ${ }^{18}$.

- Utilización sostenible de las superficies forestales. La silvicultura forma parte integrante del desarrollo rural y la ayuda a la utilización sostenible de las tierras debe incluir la gestión sostenible de los bosques y su papel multifuncional. En este contexto, se concederán ayudas a la primera forestación de tierras no agrícolas, la primera implantación de sistemas agroforestales en tierras agrícolas, ayudas Natura 2000, ayudas en favor del medio forestal, acciones para recuperar el potencial forestal y medidas preventivas e inversiones no productivas relacionadas con las intervenciones en favor del medio ambiente silvícola.

17 Directiva 409/79 del Consejo, de 2 de abril, relativa a la conservación de las aves silvestres (DO. L 103 de 25.04.1979).

http://eur-lex.europa.eu/LexUriServ/site/es/consleg/1979/L/01979L0409-20070101-es.pdf

Directiva 43/92 del Consejo, de 21 de mayo, relativa a la conservación de los hábitats naturales y de la fauna y la flora silvestres (DO. L 206 de 22.7.1992).

http://eur-lex.europa.eu/LexUriServ/site/es/consleg/1992/L/01992L0043-20070101-es.pdf

18 Directiva 60/2000 del Parlamento Europeo y del Consejo, de 23 de octubre, por la que se establece un marco comunitario de actuación en el ámbito de la política de aguas (DO. L 327 de 22.12.2000). http://eur-lex. europa.eu/LexUriServ/site/es/consleg/2000/L/02000L0060-20011216-es.pdf 


\subsubsection{Medidas del Eje 3}

$\mathrm{Su}$ objetivo fundamental es ofrecer un «espacio rural vivo» y contribuir a mantener y mejorar el tejido socioeconómico, en particular en el denominado rural profundo sometido al imparable fenómeno de la despoblación. Para aumentar el atractivo de las zonas rurales es necesario promover el crecimiento sostenible y generar nuevas oportunidades de empleo, en particular para mujeres y jóvenes, mejorar el acceso a servicios e infraestructuras básicas así como el acceso a las nuevas tecnologías de la información y la comunicación.

Para ello se incluyen medidas ligadas a la:

- Diversificación de la economía rural a través de ayudas para el desarrollo de actividades no agrícolas, ayuda a la creación y el desarrollo de pequeñas empresas y el fomento de actividades turísticas.

- Mejora de la calidad de vida en las zonas rurales a través del establecimiento de servicios básicos para la economía y la población rural, incluidas las actividades culturales y de ocio, en un pueblo o grupo de pueblos e infraestructuras a pequeña escala así como la conservación y mejora del patrimonio rural.

- Formación de la población rural así como adquisición de capacidades y promoción a través de estudios y actividades de información a cerca de la zona en cuestión y de la formación del personal que participa en la preparación y aplicación de la estrategia de desarrollo local.

\subsubsection{El Eje Leader}

El modelo Leader debe consolidarse a nivel de la UE integrando lo que era una Iniciativa Comunitaria como un elemento obligatorio de los Programas de Desarrollo Rural que deben aplicar los Estados miembro durante el período 2007-2013. Se financia:

- La aplicación de las estrategias de desarrollo local de los Grupos de Acción Local basadas en uno o más de los tres Ejes temáticos.

- Los proyectos de cooperación (transnacional e interterritorial) entre estos grupos.

- Los costes operativos de los GAL, la elaboración de las estrategias de desarrollo local y las actividades de promoción del territorio.

\subsection{Aplicación y fondos para dicha política}

Se creará una Red de desarrollo rural a nivel nacional y comunitario con objeto de mejorar todos los aspectos relativos a la aplicación, evaluación e intercambio de mejoras prácticas. Cada Estado miembro establecerá una red rural nacional que reunirá a las organizaciones y administraciones participantes en el desarrollo rural. Llevarán a cabo, por un lado, la identificación y el análisis de las mejores prácticas, facilitando información sobre ellas y organizando los intercambios de experiencias y de conocimientos y, por otro, prepararán programas de formación para los Grupos de Acción Local en vías de constitución y prestarán asistencia técnica para la cooperación interterritorial y transnacional entre los GAL. La Red Europea de Desarrollo Rural reunirá a las redes nacionales, las organizaciones y las administraciones activas en el sector del desarrollo rural a nivel comunitario y los objetivos y tareas son similares a las nacionales. 
La contribución financiera de la UE al desarrollo rural se adaptará a una programación plurianual regulada en el Reglamento 1290/2005 sobre la financiación de la Política Agrícola Común ${ }^{19}$. En él se crean dos nuevos fondos para 2007:

- El Fondo Europeo Agrícola de Garantía (FEAGA) que financia el primer pilar.

- El Fondo Europeo Agrícola de Desarrollo Rural (FEADER) que financia el segundo.

Por lo que a la cuantía se refiere, en su reunión de diciembre de 2005, el Consejo Europeo acordó las nuevas Perspectivas Financieras para el período 2007-2013 (Consejo Europeo, 2006). En este marco, se asignó al desarrollo rural la cantidad de $69.750 \mathrm{M€}$. La Comisión aprobó en septiembre de 2006 una Decisión ${ }^{20}$ por la que se fija el presupuesto anual para el período 2007-2013, que asciende a un total de $77.660 \mathrm{M€}$, ya que incluye la modulación obligatoria para los pagos del primer pilar (4 \% en 2007 y $5 \%$ del 2007 en adelante) y las transferencias del algodón y del tabaco. La Decisión incluye una subdivisión en asignaciones presupuestarias por año y por Estado miembro. A fin de tener en cuenta la adhesión de Bulgaria y de Rumania, esta Decisión se ha modificado el 1 de junio de 2007 para incluir las asignaciones respectivas de estos dos países con lo que la cifra global para todo el periodo asciende a 88.294 $\mathrm{M€}^{21}$. Por último, la cantidad final se ha vuelto a modificar en octubre de 2007 para incluir la modulación facultativa aplicada por el Reino Unido hasta los $90.983 \mathrm{M}^{22}$.

El Consejo Europeo también decidió que los Estados miembro, a discreción de éstos, podrían transferir sumas adicionales de los pagos directos hasta un porcentaje máximo del $20 \%$ para destinarlos al desarrollo rural.

Los porcentajes de cofinanciación de la UE fijados para cada Eje no podrán ser inferiores al $20 \%$ ni superiores al $50 \%$ (75 \% en las regiones de Convergencia). En el caso del Eje 2 y del Eje Leader, el porcentaje máximo será del $55 \%$ (80 \% en las regiones de Convergencia), reflejando la prioridad comunitaria atribuida a estos Ejes. En el caso de las regiones ultraperiféricas y de las islas del mar Egeo, el porcentaje máximo de cofinanciación podrán aumentarse hasta el $85 \%$.

El reparto por países se recoge en el cuadro $n^{\circ} 2$ y en la figura $n^{\circ} 2$. La primera cuestión a destacar es que los países adherentes recibirán un 40\% de los fondos de la PDR mientras que el $60 \%$ irá a la Europa de los 15. Con respecto a nuestro país, se le han asignado 7.214 M€ lo que supone el 7,93\% del total. Esta cantidad nos sitúa como quinto perceptor de fondos dentro de la UE a 27 miembros, muy alejado de Polonia, que casi nos duplica en asignación — 13.230 M€ lo que supone el 14,54\% - pero muy cercanos a Italia, Alemania y Rumanía que oscilan en torno al 9\% y los 8.000 M€. La segunda cuestión es que la

19 Reglamento (CE) n 1290/2005 del Consejo, de 21 de junio, sobre la financiación de la política agrícola común (DO. L 209 de 11.8.2005).

http://eur-lex.europa.eu/LexUriServ/site/es/consleg/2005/R/02005R1290-20070412-es.pdf

20 Decisión de la Comisión, de 12 de septiembre de 2006, por la que se fija el desglose anual por Estados miembro de la ayuda comunitaria al desarrollo rural en el período comprendido entre el 1 de enero de 2007 y el 31 de diciembre de 2013 (DO. L 261 de 22.9.2006).

http://eur-lex.europa.eu/LexUriServ/LexUriServ.do?uri=OJ:L:2006:261:0032:0034:ES:PDF

21 Decisión de la Comisión, de 1 de junio de 2007, por la que se fija el desglose anual por Estado miembro de la ayuda comunitaria al desarrollo rural en el período comprendido entre el 1 de enero de 2007 y el 31 de diciembre de 2013 (DO. L 142 de 5.6.2007).

http://eur-lex.europa.eu/LexUriServ/site/es/oj/2007/1_142/1_14220070605es00210022.pdf

22 Decisión de la Comisión, de 22 de octubre de 2007, por la que (...) (DO. L 280 de 24.10.2007).

http://eur-lex.europa.eu/LexUriServ/LexUriServ.do?uri=OJ:L:2007:280:0027:0030:ES:PDF 
Cuadro $\mathrm{n}^{\mathrm{o}} 2$.

REPARTO DE LOS FONDOS PARA LA POLÍTICA DE DESARROLLO RURAL. 2007-2013

\begin{tabular}{|c|c|c|}
\hline Países & Euros & $\%$ \\
\hline Alemania & 8.112 .517 .055 & 8,92 \\
\hline Austria & 3.911 .469 .992 & 4,30 \\
\hline Bélgica & 418.610 .306 & 0,46 \\
\hline Bulgaria & 2.609.098.596 & 2,87 \\
\hline Chipre & 162.523 .574 & 0,18 \\
\hline Dinamarca & 444.660 .796 & 0,49 \\
\hline Eslovaquia & 1.969 .418 .078 & 2,16 \\
\hline Eslovenia & 900.266 .729 & 0,99 \\
\hline España & 7.213.917.799 & 7,93 \\
\hline Estonia & 714.658 .855 & 0,79 \\
\hline Finlandia & 2.079.932.907 & 2,29 \\
\hline Francia & 6.441 .965 .109 & 7,08 \\
\hline Grecia & 3.707 .304 .424 & 4,07 \\
\hline Hungría & 3.805 .843 .392 & 4,18 \\
\hline Irlanda & 2.339.914.590 & 2,57 \\
\hline Italia & 8.292 .009 .883 & 9,11 \\
\hline Letonia & 1.041 .113 .504 & 1,14 \\
\hline Lituania & 1.743 .360 .093 & 1,92 \\
\hline Luxemburgo & 90.037 .826 & 0,10 \\
\hline Malta & 76.633 .355 & 0,08 \\
\hline Países Bajos & 486.521 .167 & 0,53 \\
\hline Polonia & 13.230 .038 .156 & 14,54 \\
\hline Portugal & 3.929.325.028 & 4,32 \\
\hline Reino Unido & 4.598 .674 .420 & 5,05 \\
\hline República Checa & 2.815 .506 .354 & 3,09 \\
\hline Rumanía & 8.022 .504 .745 & 8,82 \\
\hline Suecia & 1.825 .647 .954 & 2,01 \\
\hline UE-15 & 53.892 .509 .256 & 59,23 \\
\hline UE-12 & 37.090 .965 .431 & 40,77 \\
\hline Total & 90.983 .474 .687 & 100,00 \\
\hline
\end{tabular}

FUENTE: Decisión 2006/410 modificada por Decisión 2007/680; Decisión 2006/636 modificada por Decisión 2007/383; Reglamento (CE) 378/2007 y Decisión 2007/679. Elaboración propia. 


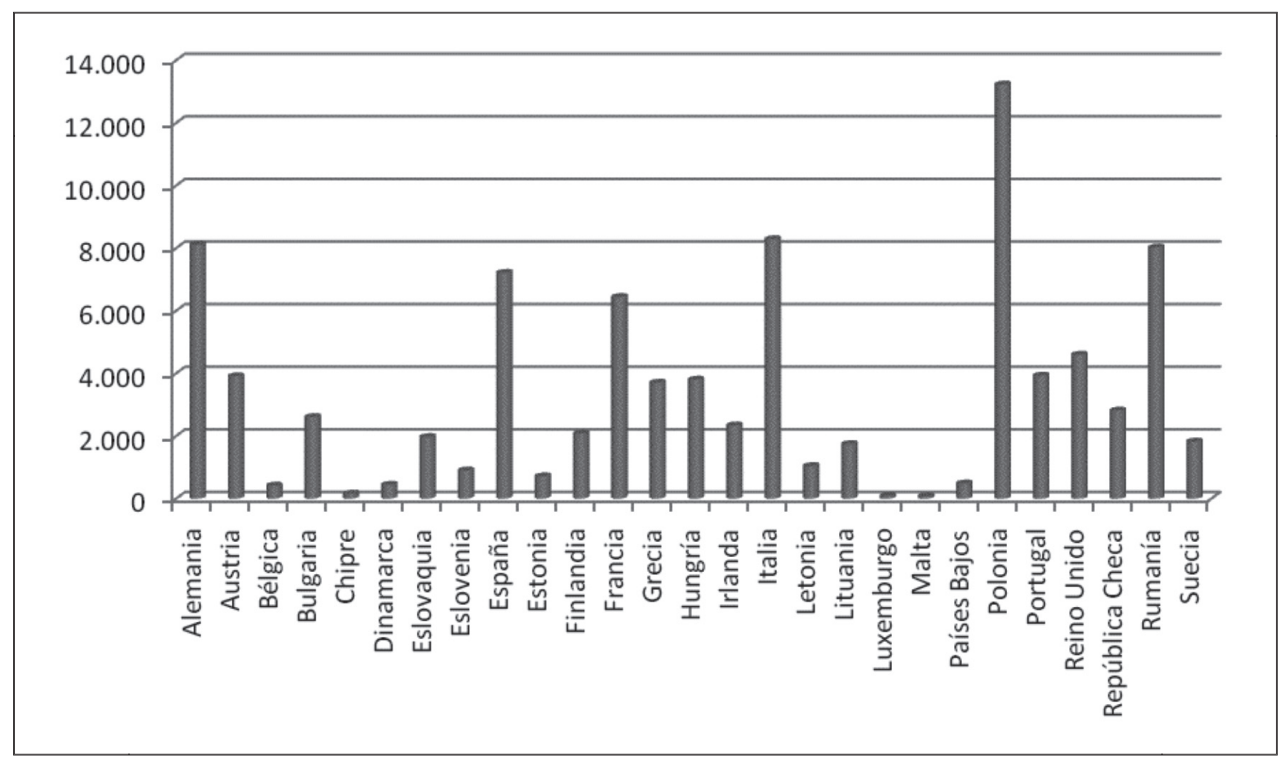

Figura No ${ }^{\circ}$. Reparto según EE.MM. de los fondos de la PDR para 2007-2013.

Fuente: Decisión 2007/680. Elaboración propia.

cantidad asignada a nuestro país es 2.000 M€ inferior a la del periodo de programación 2000-2006, recorte que ha obligado a la administración nacional a realizar una aportación adicional significativa, más allá de la obligada cofinanciación, para mantener el nivel de gasto público respecto del anterior periodo.

Pero existen otras cuestiones que debemos tener presente en relación con los fondos de los que dispondrá esta política. Para ello remitimos al cuadro $\mathrm{n}^{\mathbf{0}} 3$. La primera es que el peso de las políticas dedicadas al desarrollo rural se incrementa en términos absolutos en torno a los 1.000 M€ cada año (a precios constantes de 2004) hasta alcanzar los 7.150 para el conjunto del periodo, si bien en 2013 sólo se destina a esta política 563 M€ más que en 2006 a repartir entre 27 países y no entre 15 como ocurría en ese año. Además gana importancia internamente dentro de la PAC al llegar a representar el $21 \%$ de ésta en 2013 —el 21,6\% para el conjunto del periodo_- frente al 19 del 2006. Este incremento de fondos tiene su origen en el trasvase del primer al segundo pilar de la PAC como consecuencia de la aplicación de la modulación de las ayudas directas ya que sin ella los fondos asignados al desarrollo rural serían todos los años inferiores al de 2006 (ver figuras 3 y 4). El aspecto más negativo del desarrollo rural es que su peso dentro del conjunto del presupuesto será para 2013 el mismo valor, porcentualmente hablando, que en 2006: 8,74\%. Bien es cierto que para el conjunto del periodo los fondos asignados representan el 9,37\%, el 1,30\% procede de la modulación. La última de las cuestiones a tener en cuenta es que todos estos cambios restrictivos que estamos comentando recaen sobre los gastos de mercado y pagos directos de los países de la UE-15. Mientras en el presupuesto de 2006 estos países tenían asignado un total de $41.347 \mathrm{M} €$ en este apartado se pasa a 31.972 en 2013. Se pasa del $34,26 \%$ del presupuesto al $25,16 \%$ en 2013 , lo que supone una disminución de cerca del $10 \%$ o, lo que viene a ser lo mismo, su reducción en un tercio. 


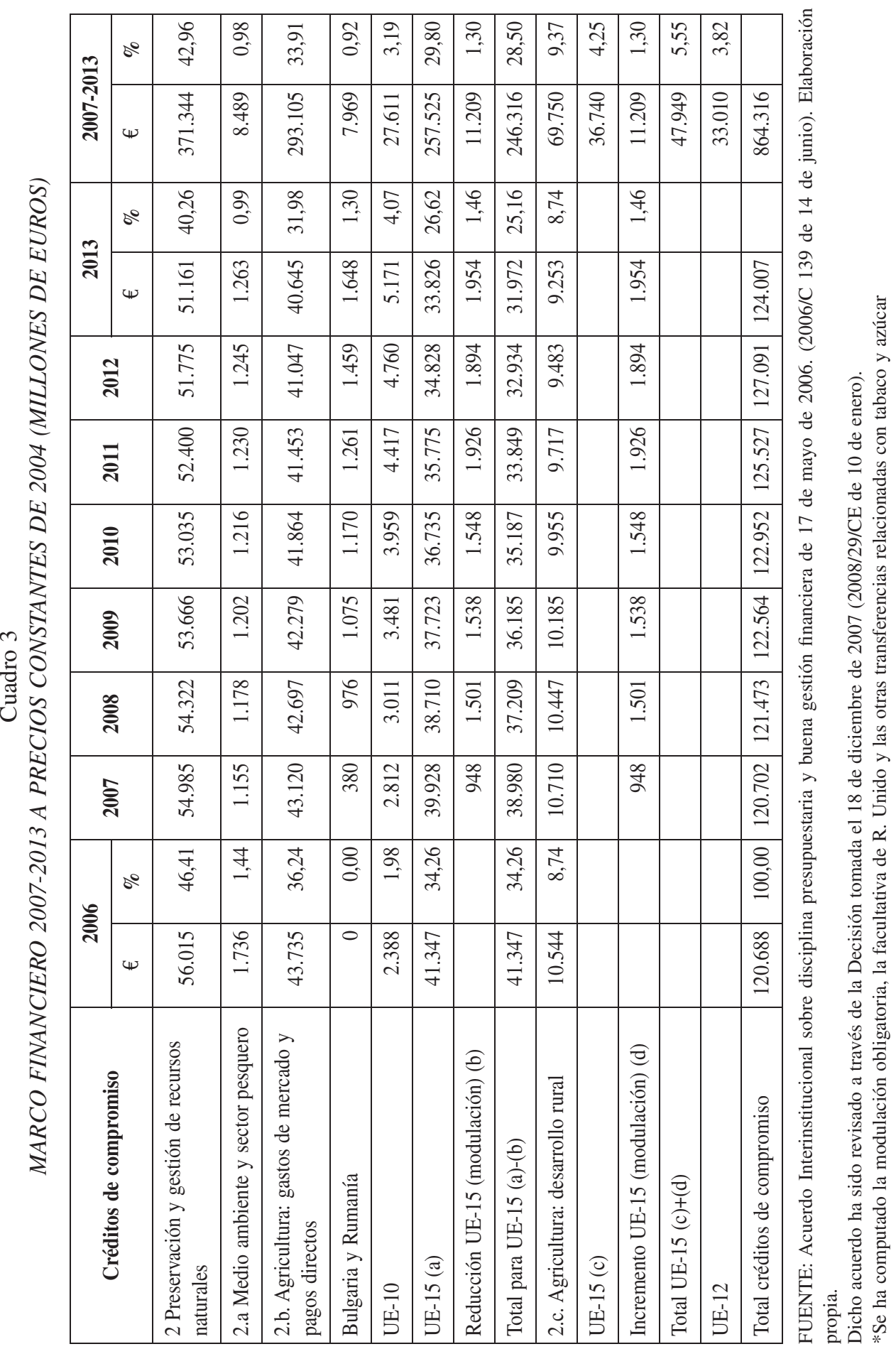




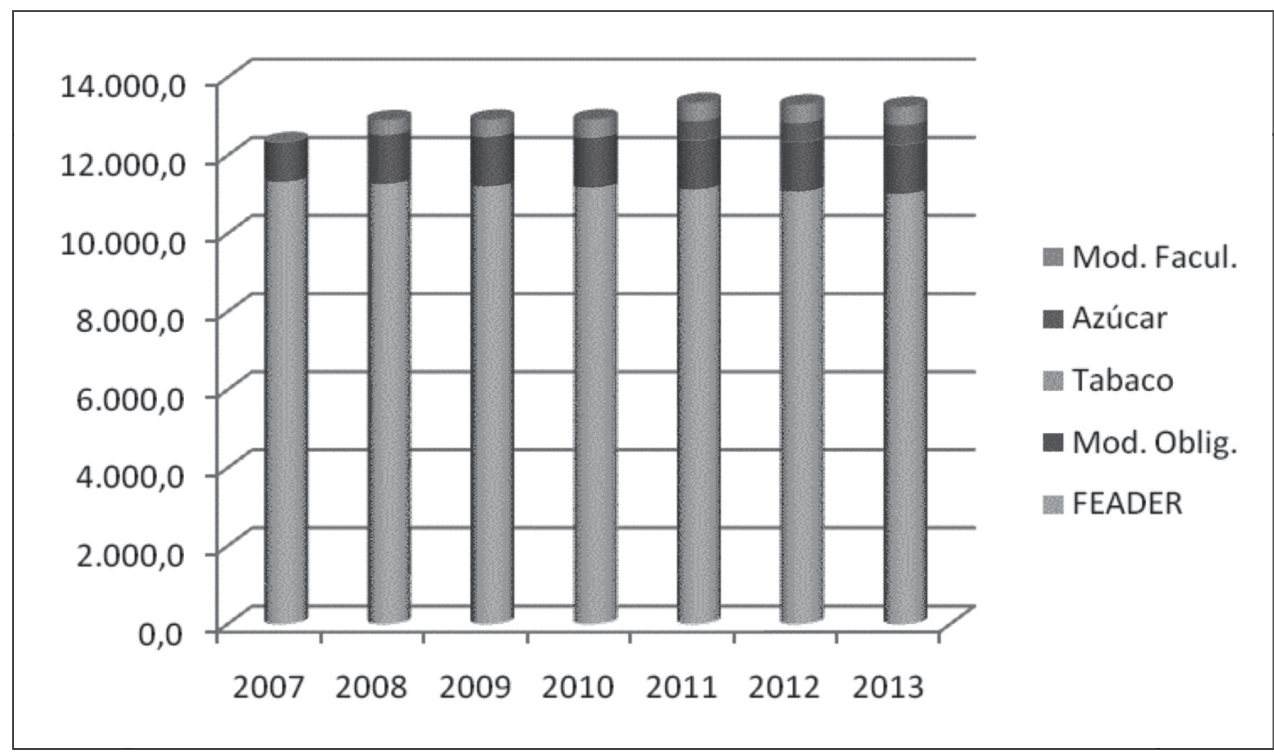

Figura $\mathbf{N}^{\mathbf{0}} 3$. Componentes de los fondos de la PDR para 2007-13 (Euros).

Fuente: Decisión 2007/383; Decisión 2007/679 y Reglamento 378/2007. Elaboración propia.

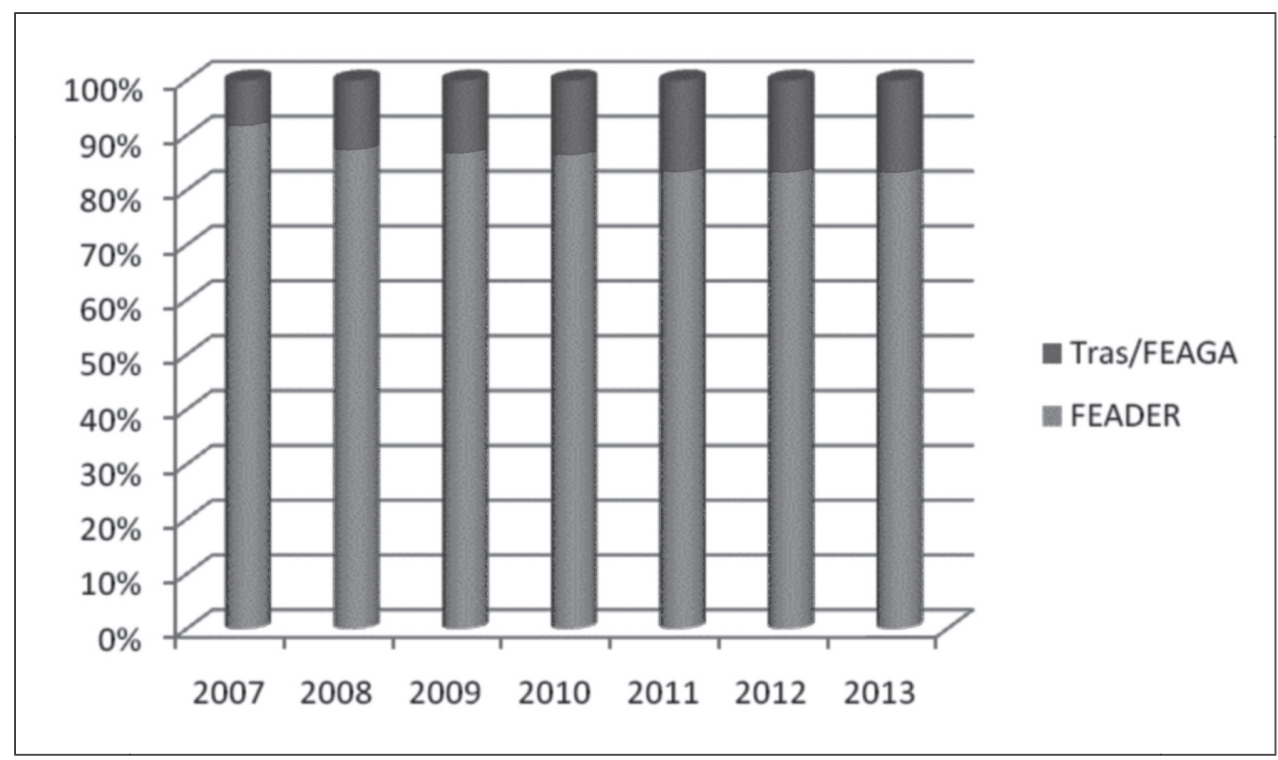

FIgura $\mathrm{N}^{\mathrm{o}}$ 4. Componentes de los fondos de la PDR para 2007-13 (\%).

Fuente: Decisión 2007/383; Decisión 2007/679 y Reglamento 378/2007. Elaboración propia. 


\subsection{Una valoración crítica}

El desarrollo rural constituye desde la aprobación de la Agenda 2.000 el segundo pilar de la PAC. La apuesta que por él viene haciendo la Comisión ha sido hasta la fecha más verbal que real, pues ni se han diseñado unas líneas directrices claras — tal y como reconoció el Tribunal de Cuentas en su Informe- ni se han aportado los recursos suficientes para que éste deje de ser la cenicienta de la PAC.

Es indudable que la nueva configuración que esta política presenta para el actual periodo de programación ofrece aspectos destacables. Algunas de las medidas ya existentes se han reforzado y se han incorporado otras como la concesión de ayudas a las explotaciones que se encuentran en zonas comprendidas dentro de la Red Natura 2.000, más recursos para la instalación de jóvenes agricultores, mayor apoyo a la aplicación de medidas agroambientales. Su estructura en base a tres Ejes temáticos y otro metodológico, a los que se le aplica una filosofía que ha demostrado su eficacia a través de Leader, dentro de unas directrices estratégicas comunes para Europa pero que permiten flexibilidad a la hora de su aplicación en los diferentes países, es también positivo. La simplificación de los procedimientos y fondos para su gestión es un avance innegable. La apuesta por el trasvase de fondos del primer al segundo pilar de la PAC a través de, como mínimo la modulación obligatoria, es —al menos - una buena declaración de intenciones y una senda que se debería recorrer hasta su fín. Por último, la inclusión de aspectos novedosos como el tratamiento especial que reciben la red Natura 2000, la mejora del bienestar de los animales, la calidad de los productos alimentarios y, especialmente, el cumplimiento de las normas de condicionalidad para el cobro de las ayudas europeas, son avances significativos (Cejudo y Maroto, 2007).

Por contra, una de las cuestiones más discutibles, a nuestro entender, del diseño de la Política de Desarrollo Rural es su consolidación como «segundo pilar» de la PAC. Es más que discutible que las actuaciones relacionadas con el mundo rural, de fuerte ligazón territorial, se hayan trasladado como un apéndice de la política sectorial por antonomasia de la UE como es la PAC. Este hecho permite afirmar que la nueva formulación del desarrollo rural se entiende más como un complemento de la política agraria de mercados y rentas que como parte del objetivo general de cohesión económica, social y territorial europea. No conviene perder de vista que la PAC está - y siempre ha estado- muy lejos de ser una política reequilibradora social y territorialmente hablando. Es cierto que pueden existir motivaciones «técnico-presupuestarias» que permiten el trasvase de fondos de un pilar a otro dentro de la misma rúbrica, pero deberían ser aquéllas las que se adecuaran al diseño general y no al contrario.

En este mismo sentido, y como consecuencia de lo que acabamos de decir, la nueva formulación del desarrollo rural adolece de la diversidad intrínseca al mundo rural europeo tal y como ocurría cuando estaba integrado, en parte, dentro de la Política Regional en la que sí se contemplaban tratamientos diferenciados entre las distintas características de cada región y de cada zona, aspecto éste que ahora sólo queda circunscrito a los Ejes 3 y 4 exclusivamente. En definitiva, el enfoque que entendemos debe ser nítidamente territorial de este tipo de políticas — reflejo del carácter multifuncional de estos espacios- superador de encorsetados enfoques sectoriales, integrador y territorial, queda claramente ensombrecido.

De no menor trascendencia es el hecho de la escasa dotación presupuestaria asignada a esta política en la medida en que aquélla refleja, bien a las claras, el interés real por abordar un problema desde las instituciones públicas. El total de fondos asignados para el programa (ver cuadro $\mathrm{n}^{\circ}$ 2) va a ser de $90.983 \mathrm{M} €$ con una UE ampliada a 27 miembros. Es evidente que con esa cantidad no se solucionan los problemas de estas zonas y supone, lo que es más 
grave, un recorte con respecto al periodo 2000-2006 en la medida en que el ligero avance global que se produce en términos presupuestarios para el conjunto de la UE se aplicará a 12 países más, a 100 millones de habitantes más y a nuevos programas como el Natura 2000.

Es más, el trasvase de fondos del primer pilar al segundo sobre la base de la modulación obligatoria se ha demostrado raquítico financieramente hablando —el 5\% establecido se aleja abismalmente del $20 \%$ fijado en la propuesta de Reglamento- y lo que es más grave insostenible socialmente en la medida en que éste es ridículo para legitimar una política pública. Difícilmente se pueden seguir sosteniendo socialmente el trasvase a manos de grandes propietarios de ingentes cantidades de dinero cuando son responsables de empresas bien dimensionadas y capitalizadas. La justificación del pago único y su legitimación social estará en la remuneración de externalidades positivas, en la producción de bienes públicos y en el cumplimiento de funciones sociales y ambientales. «Hay que pasar del acoplamiento a la producción al acoplamiento al territorio» (Moyano, E., 2007, 18). La Comisión intenta contrarrestar dicha situación a través, primero, de la propuesta del incremento de los fondos dedicados al Desarrollo Rural a través de un aumento del $2 \%$ de la modulación obligatoria para 2010-2013, estudiándose su posible aplicación a los países recientemente incorporados que se encuentran exentos de ella. Segundo, de la introducción de la degresividad en las ayudas agrarias, tal y como se recoge en la Comunicación que anuncia el «chequeo médico» de la PAC de este año (Comisión, 2007a).Tercero, simplificando los requisitos y procedimientos en el cumplimiento de la condicionalidad (Comisión, 2007b). Iniciativas que quedan ensombrecidas ante datos tan aplastantes como los que ofrece el Tribunal de Cuentas en relación al cobro de las ayudas de la PAC $(2007,123)$. Afirma que se han incorporado nuevos beneficiarios entre los que destacan «las compañías de ferrocarriles, los clubs de equitación/cría de caballos, los clubs de golf/ocio y los ayuntamientos en el Reino Unido, Alemania, Suecia y Dinamarca. La asignación de derechos a propietarios que no han ejercido nunca la actividad agrícola y que simplemente conservan las tierras en buenas condiciones agrarias y medioambientales ha dado lugar a una redistribución importantes de las ayudas comunitarias que han pasado de los agricultores a los propietarios».

Otro aspecto a tener en cuenta es el conflicto que, a varios niveles, ha generado y lo va a seguir haciendo este nuevo diseño. Este conflicto de intereses no sólo se sustenta en el hecho de que los actores beneficiados por estos programas sean diferentes en su procedencia, intereses, organizaciones que los representan, etc., en lucha por su gestión y la captación de los fondos asignados sino también porque la filosofía que está detrás de cada uno de los diferentes Ejes es tan dispar que se nos aventura bastante difícil su integración.

En efecto, en primer lugar, se enfrentan los agricultores y las organizaciones que los representan frente a otros posibles beneficiarios por unos fondos escasos y que además, en parte, proceden del primer pilar de la PAC, a través de la modulación de la ayudas, en evidente retroceso presupuestario. A ello se añaden los recelos existentes a la hora de gestionar y repartir los fondos por parte de los GAL y de las organizaciones agrarias, de histórica implantación y amplias relaciones con las administraciones públicas, ahora obligadas a convivir. Estas tensiones se resolvieron sin la victoria completa de ninguna de las partes optándose por lo que ha sido denominado como desarrollo rural «agro-territorial» (Compés, 2005: 56). En segundo lugar, la búsqueda de la competitividad en el sector agrario contemplada en el primer Eje y dirigida a los agricultores casa mal, a priori, con la necesidad de sostenibilidad del segundo del que se beneficiarán no sólo agricultores (ayudas medioambientales) sino también propietarios forestales a través de Natura 2000 (autóctonos de la zona o no, dedicados al sector o no, comprometidos con esta estrategia de desarrollo rural o no) y ambos con la creación de empleo y la mejora de la calidad de vida en la que se encuentran implicados todos los demás actores sociales tanto del mundo rural como del 
urbano en la medida en que la financiación de infraestructuras básicas, equipamientos y servicios públicos y privados, etc., no compete sólo al mundo rural. En tercer lugar, todo ello debe desarrollarse, necesariamente, a través de una metodología, puesta en marcha por la Iniciativa Leader y los GAL, que se articula mal con la llevada a cabo por parte de las administraciones e instituciones públicas y privadas ahora involucradas en este nuevo diseño. Finalmente, está por ver si los GAL son «capaces de ir más allá de una simple estructura de gestión de fondos públicos como ha sido Leader y Proder; sobre todo tienen que superar la concepción y/o visión entre la comunidad local como un conjunto de actores más interesados en utilizarlos como instrumento de poder, de influencia en las comunidades locales o de proyección personal en el ámbito de las organizaciones de carácter político, económico o social» (Esparcia, 2006: 89).

La perspectiva amplia del desarrollo rural en la que se inspira el nuevo Reglamento plantea también importantes retos sociales e institucionales. Uno de ellos es el de la creación de nuevas agencias públicas capaces de coordinar las distintas actuaciones administrativas y de crear marcos adecuados de concertación con los actores sociales y económicos implicados. Hay quienes alertan ya de los riesgos de seguir canalizando las nuevas políticas de desarrollo rural a través de los ministerios o departamentos de Agricultura y Medio Ambiente, sobre todo si se tiene en cuenta que estas nuevas políticas parten de una concepción del mundo rural distinta de la que caracteriza a esos departamentos. Otro, la necesidad de establecer un «pacto social» que implique no sólo a los sectores agrarios, ni tan siquiera a los demás agentes rurales, sino al conjunto de la sociedad rural-urbana, urbana-rural. Somos conscientes que es más fácil decirlo que hacerlo pero lo segundo no invalida la necesidad de lo primero.

Dos cuestiones más para terminar. De un lado, nos queda la duda, más que razonable por otra parte como lo avala el devenir histórico en el diseño de estos programas, de la continuidad temporal del nuevo diseño así como de su planificación y financiación. De otro, la necesaria coordinación con otras políticas de carácter territorial, tanto a nivel comunitario como nacional, plantea la necesidad para nuestro país de acometer políticas nacionales y regionales propias y complementarias de las europeas ligadas al desarrollo rural y territorial. La «Ley para el desarrollo sostenible del medio rural» recientemente aprobada en nuestro país afirma que «es una Ley de orientación territorial, lo que implica que será aplicada tomando en consideración criterios y directrices de ordenación territorial. Singularmente, la Ley contempla zonas rurales diferenciadas según una tipología establecida, define zonas rurales prioritarias y prevé el establecimiento de un plan por zona rural. Las acciones $y$ medidas previstas en la Ley son multisectoriales y medioambientales. Coherentemente, reflejan la nueva realidad de un medio rural económicamente cada vez más diversificado y al que se le reconoce una importante multifuncionalidad para la sociedad en su conjunto». Esta declaración de intenciones es un buen comienzo que necesita, para ser creída, concretarse en diseños y actuaciones concretas. Pero eso está, lógicamente, por desarrollar por el nuevo Ministerio de «Medio Ambiente, Medio Rural y Marino» en el que se han integrado las competencias medio ambientales y las del antiguo Ministerio de Agricultura.

\section{Bibliografía y documentación utilizada. (La normativa se incluye a pie de página)}

ALARIO TRIGUEROS, M. y BARAJA RODRÍGUEZ, E. (2006): «Políticas públicas de desarrollo rural en Castilla y León. ¿sostenibilidad consciente o falta de opciones?: LEADER II» en Boletín de la AGE, nº. 41, pp. 267-294.

ARMESTO LÓPEZ, X.A. (2007): «El concepto de agricultura ecológica y su idoneidad para fomentar el desarrollo rural sostenible», en Boletín de la AGE, nº 43, p. 155-172. 
CEJUDO GARCÍA, E. y MAROTO MARTOS, J.C. (2000): «La política territorial en Andalucía. Los Programas Operativos Territoriales». Revista de Estudios Regionales, $\mathrm{n}^{\circ}$ 56, pp. 117-139.

CEJUDO GARCÍA, E. (2001): «Los desequilibrios territoriales de la Política Agraria Comunitaria». Cuadernos Geográficos de la Universidad de Granada, $\mathrm{n}^{\circ}$. 30, pp. 143-164.

CEJUDO GARCÍA, E. y NAVARRO VALVERDE, F. (2003): «El reparto territorial de los Programas de Desarrollo Rural. El caso de la provincia de Granada» en Anales de Geografía de la Universidad Complutense, $\mathrm{n}^{\circ}$. 23, pp. 131-162.

CEJUDO GARCÍA, E., Y MAROTO MARTOS, J.C. (2007): «La importancia del Patrimonio en la Política de Desarrollo Rural de Andalucía». e-rph.. Revista electrónica de Patrimonio Histórico, 1. Granada, pp. 243-280. http://www.revistadepatrimonio.es/revistas/numero1/patrimonio/estudios/articulo.php

COMISIÓN EUROPEA (1997): Agenda 2000: por una Unión más fuerte y más amplia. COM (97) 2000 final.

COMISIÓN EUROPEA (2004a): Propuesta de Reglamento del Consejo relativo a la financiación de la política agrícola común. COM (2004) 489 final de 14 de julio.

http://eur-lex.europa.eu/LexUriServ/LexUriServ.do?uri=COM:2004:0489:FIN:ES:PDF

COMISIÓN EUROPEA (2004b): Propuesta de Reglamento del Consejo relativo a la ayuda al desarrollo rural a través del FEADER. COM (2004) 490 final de 14 de julio. http://eur-lex.europa.eu/LexUriServ/LexUriServ.do?uri=COM:2004:0490:FIN:ES:PDF

COMISIÓN EUROPEA (2005): Actualización del informe de la Evaluación de Impacto Ampliada adjunta a la propuesta de Reglamento del Consejo relativo al apoyo al desarrollo rural. SEC (2005) 914.

http://ec.europa.eu/transparency/regdoc/rep/2/2005/EN/2-2005-914-EN-1-0.Pdf

COMISIÓN EUROPEA (2007a): Preparándose para el «chequeo» de la reforma de la PAC. COM (2007) 722 final de 20 de noviembre. http://eur-lex.europa.eu/LexUriServ/LexUriServ.do?uri=COM:2.007:0722:FIN:ES:PDF

COMISIÓN EUROPEA (2007b): Informe sobre la aplicación del sistema de condicionalidad. COM (2007) 147 final de 29 de marzo.

http://eur-lex.europa.eu/LexUriServ/LexUriServ.do?uri=COM:2.007:0147:FIN:ES:PDF

COMISIÓN EUROPEA (2008): Les Européenns, l'agriculture et la politique agricole commune en 2007. Eurobarométre Spécial. Bruselas.

http://ec.europa.eu/agriculture/survey/fullreport_fr.pdf

COMPÉS LÓPEZ, R. «Los principios de la reforma «intermedia». En: GARCÍA ÁLVAREZ-COQUE, J.M y Otros (2005): La reforma de la Política Agraria Común. Madrid. Ed. Eumedia y MAPA, pp. 35-60.

CONFERENCIA EUROPEA SOBRE EL DESARROLLO RURAL (1996): Declaración de Cork. Por un medio rural vivo. Celebrada en Cork (Irlanda) entre el 4 y 9 de noviembre. http://ec.europa. eu/ agriculture/rur/cork_fr.htm.

CONFERENCIA EUROPEA DE DESARROLLO RURAL (2003): Semer les graines du monde rural de demain. Les perspectives de la politique rurale dans une Europe élargie. Salzburgo. 12-14 de noviembre de 2003.

http://ec.europa.eu/agriculture/events/salzburg/proceedings1_fr.pdf

CONSEJO EUROPEO (2000): Conclusiones de la Presidencia del Consejo Europeo de Lisboa de 23 y 24 de marzo.

http://www.consilium.europa.eu/ueDocs/cms_Data/docs/pressData/es/ec/00100-r1.es0.htm

CONSEJO EUROPEO (2001): Conclusiones de la Presidencia del Consejo Europeo de Gotemburgo de 15 y 16 de junio.

http://www.consilium.europa.eu/ueDocs/cms_Data/docs/pressData/es/ec/00200-r1.es1.pdf

CONSEJO EUROPEO (2006): Conclusiones de la Presidencia del Consejo Europeo de Bruselas de 15 y 16 de diciembre.

http://www.consilium.europa.eu/ueDocs/cms_Data/docs/pressData/es/ec/87658.pdf

El documento que las contienen es el 15915/05.

http://register.consilium.europa.eu/pdf/es/05/st15/st15915.es05.pdf 
CONSEJO DE LA UNIÓN EUROPEA (2006): Directrices Estratégicas comunitarias de desarrollo rural para el periodo de programación 2007-2013. Decisión (CE) nº 144 de 20 de febrero (DO. L 55 de 25.2.2006). http://eur-lex.europa.eu/LexUriServ/site/es/oj/2006/1_055/1_05520060225es00200029.pdf

DELGADO SERRANO, M.D. (2004): La política rural europea en la encrucijada. MAPA. Madrid. $350 \mathrm{pp}$.

DÍEZ PATIER, E. y TRUEBA HERRANZ, D. (2006): «Evolución del uso del término multifuncionalidad en el contexto europeo». En: GÓMEZ-LIMÓN, J.A. Y BARREIRO HURLÉ, J. (Coords): La multifuncionalidad de la agricultura en España. Concepto, aspectos horizontales, cuantificación y casos prácticos. EUMEDIA, S.A. y MAPA. Madrid, pp. 41-56.

ESPARCIA, J., NOGUERA, J., FERRER, M. (2003) «La innovación empresarial y la difusión como nuevos factores de desarrollo territorial. Una comparación entre dos áreas geográficas de diferente accesibilidad» en Boletín de la AGE, $n^{o}$ 36, p. 149-160.

ESPARCIA PÉREZ, J. (2006): «Leader II y Proder I en el desarrollo rural en España». En: FRUTOS MEJÍAS, L.M Y RUÍZ BUDRÍA, E. (Eds): Estrategias territoriales de desarrollo rural. Excma. Diputación de Zaragoza. Zaragoza, p. 65-90.

ETXEZARRETA, M. et Al. (2005): Por una política democrática frente al dominio de los mercados. Propuestas para una estrategia integrada de desarrollo en Europa. http://www.fpereardiaca.org/llibres/Euromemorandum_2005.pdf

ETXEZARRETA, M. (Coord) (2006): La agricultura española en la era de la globalización. MAPA. Madrid. $751 \mathrm{pp}$.

MÁRQUEZ FERNÁNDEZ, D. et AL (2006): «El precio de la sostenibilidad rural en Andalucía: el valor de Leader II» en Boletín de la AGE, n 41, pp. 295-314.

MINISTERIO DE AGRICULTURA PESCA Y ALIMENTACIÓN. (2002): El futuro del mundo rural. MAPA. Madrid.

MINISTERIO DE AGRICULTURA PESCA Y ALIMENTACIÓN. (2003): Libro Blanco de Agricultura y Desarrollo Rural. MAPA. Madrid, 3 Tomos.

MOLINERO HERNANDO, F. (2002): «El desarrollo rural en los territorios de la Unión Europea». En: Jornadas Temáticas sobre El Mundo Rural. MAPA. Madrid 23 de mayo. 20 pp.

MOLINERO HERNANDO, F. (2006): «La evolución de la agricultura en España: tradición, modernización y perspectivas». Norba. Revista de Geografía. Cáceres, Vol. XI, págs. 85-106.

MOLLARD, A. (2003): «Multifonctionalité de lágriculture et territoires: des concepts aux politiques publiques». Cahiers d'économie et sociologie rurales, $\mathrm{n}^{\circ}$ 66, pp. 28-54.

MOLTÓ MANTERO, E. y HERNÁNDEZ HERNÁNDEZ, M. (2004): «La funcionalidad de los medios rurales en las sociedades urbanas». Investigaciones Geográficas, n 34, p. 63-76.

MOYANO ESTRADA, E. y GARRIDO FERNÁNDEZ, F.E. (2006): «A propósito de la multifuncionalidad». En: GÓMEZ-LIMÓN, J.A. Y BARREIRO HURLÉ, J. (Coords): La multifuncionalidad de la agricultura en España. Concepto, aspectos horizontales, cuantificación y casos prácticos. EUMEDIA, S.A. y MAPA, Madrid, pp. 56-76.

MOYANO ESTRADA, E. (2007): «Sobre agricultura y política. Reflexiones en un periodo de transición». En: AA.VV: Agricultura familiar en España 2007. Fundación de Estudios Rurales, Madrid, pp. 5-7.

NAVARRO VALVERDE, F. (2007): Repercusión de los programas de desarrollo rural en la provincia de Granada. Leader I, II y Proder I. Universidad de Granada. Granada. Tesis Doctoral (en prensa).

OCDE. (2006): El nuevo paradigma rural. Políticas y gobernanza. OCDE y MAPA. Madrid. 186 pp.

PLAZA GUTIERREZ, J.I. (2006): «Territorio, Geografía rural y políticas públicas. Desarrollo y sustentabilidad en las áreas rurales» en Boletín de la AGE, $\mathrm{n}^{\circ}$ 41, p. 69-98.

SÁENZ LORITE, M. CEJUDO GARCÍA, E. MAROTO MARTOS, J.C. (2000): «Una Iniciativa Comunitaria para el desarrollo rural. Análisis del Leader I y II en Andalucía». En: Lecturas geográficas. Homenaje a José Estébanez Álvarez. Editorial Complutense. Madrid. Vol. I, pp. 899-916.

TRIBUNAL DE CUENTAS. (2007): Informe anual relativo al ejercicio 2006. http://eca.europa. eu/portal/pls/portal/docs/1/481525.PDF 